\title{
SOME REMARKS ON A CLASS OF MATRIX INEQUALITIES ${ }^{1}$
}

\author{
S. G. WILLIAMSON
}

1. Introduction. In this note we consider a class of matrix functions associated with finite permutation groups and their representations. We shall show that, for an extensive class of such functions, equality of the given function with the determinant in the case of positive definite hermitian matrices can occur if and only if the matrix is diagonal. We also partially characterize the case of equality in a more general class of inequalities [1].

Let $G$ be a finite permutation group acting on $Z^{n}=[1, \cdots, n]$ and let $M$ denote a representation of $G$ as unitary linear operators on an $m$-dimensional unitary space $U$. Let $D$ be an $n \times n$ matrix. We consider

$$
\begin{aligned}
M_{D} & =\sum_{\sigma \in G} M(\sigma) \prod_{t=1}^{n} d_{t \sigma(t)} \\
d_{\chi}(D) & =\operatorname{tr}\left(M_{D}\right)=\sum_{\sigma \in G} \chi(\sigma) \prod_{t=1}^{n} d_{t \sigma(t)} .
\end{aligned}
$$

( $\chi$ denotes the character of $M$.)

The "determinant" and "permanent" are, of course, special cases of (1) and (2). It is known that the det $H=$ per $H$ if and only if $H$ is diagonal where $H$ is positive definite hermitian [2]. In general,

$$
d_{\chi}(H) \geqq m \operatorname{det} H
$$

for $H$ positive semidefinite hermitian.

Inequality (3) is a result of I. Schur [3] and is a special case of an inequality of M. Marcus [1]:

$$
\left|\left(M_{A B} x, y\right)\right|^{2} \leqq\left(M_{A A^{*}} x, x\right)\left(M_{B^{*} B} y, y\right)
$$

where $A$ and $B$ are any two $n$-square matrices and $x$ and $y$, any two vectors in $U$.

2. Main results. Let $J$ denote the matrix with all entries 1 . Then

$$
M_{J}=\sum_{\sigma \in G} M(\sigma)
$$

Received by the editors February 13, 1967.

1 This research was supported in part by NSF Grant GP-5870. 
One may easily verify that $M_{J}$ (often denoted by $\operatorname{Tr}_{G}$ ) is hermitian and that $M_{J}^{2}=g M_{J}$ where $g$ is the order of $G$. Thus $M_{J}$ has eigenvalues $g$ or 0 .

THEOREM 1. Let $x$ and $y$ be any two vectors in $U$ such that $M_{J} x \neq 0$ and $M_{J} y \neq 0$. If $A$ and $B$ are $n$-square matrices, then

$$
\left|\left(M_{A B} x, y\right)\right|^{2}=\left(M_{A A^{*}} x, x\right)\left(M_{B^{*} B} y, y\right) \neq 0
$$

only if $M_{\mathrm{J}} x$ and $M_{\mathrm{J}} y$ are collinear and the rows of $A$ are equal, up to a permutation and scalar factors, to the rows of $B^{*}$. Both sides of (6) reduce to zero if and only if either $A$ or $B^{*}$ has a zero row.

Theorem 2. If $H$ is positive semidefinite hermitian and $x \in U$ $\|x\|=1$, and if $M_{J} x \neq 0$, then

$$
\operatorname{det} H=\left(M_{H} x, x\right) \neq 0
$$

if and only if $H$ is diagonal and nonsingular. Both sides of (7) reduce to zero if and only if $H$ has a zero row.

THEOREM 3. If $H$ is positive semidefinite hermitian and if $M_{J} x \neq 0$ for some unit eigenvector $x$ corresponding to the minimum eigenvalue of $M_{H}$, then

$$
m \operatorname{det} H=d_{\chi}(H) \neq 0
$$

if and only if $H$ is diagonal and nonsingular. Also $m$ det $H=d_{x}(H)=0$ if and only if $H$ has a zero row ( $m$ is the degree of the representation).

We remark that in the case $M(\sigma) \equiv I$ (as in the case of the permanent) $M_{J} x=g x \neq 0$ is always satisfied for $x \neq 0$ ( $g$ is the order of $\left.G\right)$. If $M(\sigma)=\epsilon(\sigma) I$ where $\epsilon(\sigma)$ is the alternating character then $M_{J} \equiv 0$ whenever $G$ contains an odd permutation. Thus Theorems 1-3 apply to "permanent type" rather than "determinant type" functions. From Theorem 3, we see that the essential assumption is that the eigenspace corresponding to the minimum eigenvalue of $M_{H}$ is not a subspace of the kernel of $M_{J}$. In the case of "permanent type" functions, $\operatorname{ker} M_{J}=\{0\}$ so this condition is always satisfied, but in the case of the "determinant type" functions, ker $M_{J}=U$, the whole space.

3. Proofs. Let $U$ and $V$ be finite-dimensional unitary spaces. Denote by $W=\otimes^{n} V(\operatorname{dim} V=s)$ the $n$th tensor product of $V$. Let $G$ and $M$ be as in $\S 1$. We define an endomorphism $T$ on $U \otimes W$ by

$$
T=\sum_{\sigma \in G} M(\sigma) \otimes P(\sigma)
$$


where $P(\sigma)\left(x_{1} \otimes \cdots \otimes x_{n}\right)=x_{\sigma^{-1}(1)} \otimes \cdots \otimes x_{\sigma^{-1}(n)}$.

One may easily verify that $T$ is hermitian (with respect to (10) below) and that $T^{2}=g T$ where $g$ is the order of $G$. Defining the natural inner product in $U \otimes V$ by

(10) $\left(u_{1} \otimes x_{1} \otimes \cdots \otimes x_{n}, u_{2} \otimes y_{1} \otimes \cdots \otimes y_{n}\right)=\left(u_{1}, u_{2}\right) \prod_{t=1}^{n}\left(x_{t}, y_{t}\right)$, and applying the Schwarz inequality to $T(x \otimes v)$ and $T(y \otimes w)$ where $x, y \in U$ and $v, w \in W\left(v=v_{1} \otimes \cdots \otimes v_{n}, w=w_{1} \otimes \cdots \otimes w_{n}\right)$ gives

$|(T(x \otimes v), T(y \otimes w))|^{2} \leqq(T(x \otimes v), T(x \otimes v))(T(y \otimes w), T(y \otimes w))$.

Using the hermitian idempotent properties of $T$ one may obtain inequality (4) by letting $V=C^{n}, v_{1}, \cdots, v_{n}$ be the rows of $A$, and $w_{1}, \cdots, w_{n}$ be the rows of $B^{*}$. For a complete proof see M. Marcus [1].

Thus the problem of determining conditions for equality in (4) is equivalent to the problem of determining conditions for the collinearity of $T(x \otimes v)$ and $T(y \otimes w)$. Thus we prove the following.

Lemma. If $v, w, M_{J} x$, and $M_{J} v$ are nonzero and

$$
T(x \otimes v)=k T(y \otimes w),
$$

then both sides of (11) are nonzero, $M_{J} x$ and $M_{J} y$ are collinear, and there exist constants $c_{1}, \cdots, c_{n}$ and a permutation $\sigma$ such that $v_{i}$ $=c_{i} w_{\sigma(i)}\left(v=v_{1} \otimes \cdots \otimes v_{n}, w=w_{1} \otimes \cdots \otimes w_{n}\right)$.

Proof. It suffices to prove the Lemma for the case $V=C^{s}$ ( $C$ is the complex numbers) with the standard inner product. Let $d=\bar{z} \otimes \cdots \otimes \bar{z}$, where $z=\left(z_{1}, \cdots, z_{s}\right)$ is in $C^{s}$, be in $W=\otimes^{n} V$ and consider $u \otimes d$ in $U \otimes W$. Then

$$
(T(x \otimes v), u \otimes d)=k(T(y \otimes w), u \otimes d)
$$

becomes

$$
\left(M_{J} x, u\right) \prod_{t=1}^{n}\left(v_{t}, \bar{z}\right)=k\left(M_{J} y, u\right) \prod_{t=1}^{n}\left(w_{t}, \bar{z}\right)
$$

where $P_{1}(z)=\prod_{t=1}^{n}\left(v_{t}, \bar{z}\right)$ and $P_{2}(z)=\prod_{t=1}^{n}\left(w_{t}, \bar{z}\right)$ are two nonzero polynomials (since $v \neq 0$ and $w \neq 0$ ) in $z_{1}, \cdots, z_{s}$. Choosing $z$ such that $P_{1}(z) \neq 0$ and $P_{2}(z) \neq 0$ and varying $u$, we see that $M_{v} x$ and $M_{J} y$ are collinear. As $M_{J} x \neq 0$ and $M_{J}$ is hermitian andidempotent, $\left(M_{J} x, x\right) \neq 0$. Again, choosing $z$ such that $P_{1}(z) \neq 0$ and setting $u=x$, we see that both sides of (12) are nonzero (and hence $k \neq 0$ and $\left(M_{J} y, x\right) \neq 0$ ). Finally, fix $u=x$ in (12). Both sides of (12) then represent the fac- 
torization of the same nonzero polynomial into linear factors. This factorization is unique up to units and order of factors. This completes the proof of the Lemma.

We remark that if either $v=0$ or $w=0$, then both sides of (11) reduce to zero.

To prove Theorem 1 we note that (6) holds if and only if $T(x \otimes v)$ $=k T(y \otimes w) \neq 0$ where $v_{1}, \cdots, v_{n}$ are the rows of $A$ and $w_{1}, \cdots, w_{n}$ are the rows of $B^{*}(s=n$ in this case). By the remark following the Lemma, if $A$ or $B^{*}$ has a zero row, then (6) cannot hold. Thus $v \neq 0$ and $w \neq 0$ and from the Lemma $M_{J} x$ and $M_{J} y$ are collinear. Also, there exist constants $c_{1}, \cdots, c_{n}$ and a permutation $\sigma$ such that $v_{i}=c_{i} w_{\sigma(i)}, i=1, \cdots, n$, so the rows of $A$ are equal, up to a permutation and scalar factors, to the rows of $B^{*}$. From the Lemma and the remark following the Lemma, both sides of (6) can reduce to zero if and only if $v=0$ or $w=0$ or, in particuiar, if and only if $A$ or $B^{*}$ has a zero row.

Theorem 2 follows if we let $H=A A^{*}$ where $A$ is triangular and let $B=I_{n}$. In this case (4) becomes (letting $x=y$ )

$$
\begin{aligned}
\left|\left(M_{A} x, x\right)\right|^{2} & =\left|\prod_{t=1}^{n} a_{t t}\right|^{2}\|x\|^{2}=|\operatorname{det} A|^{2} \\
& =\operatorname{det} H \leqq\left(M_{H} x, x\right)\|x\| \\
& =\left(M_{H} x, x\right)
\end{aligned}
$$

or $\operatorname{det} H \leqq\left(M_{H} x, x\right)$. Thus by Theorem 1 if $\operatorname{det} H=\left(M_{H} x, x\right) \neq 0$, then the rows of $A$ must be, up to order and nonzero multiples, the rows of $I_{n}$. Since $A$ is triangular, this implies that $A$ and $A A^{*}=H$ are diagonal. Conversely, if $H$ is diagonal and nonsingular, then $M_{H}$ $=(\operatorname{det} H) I_{m}$ so $\operatorname{det} H=\left(M_{H} x, x\right) \neq 0$. The case $m \operatorname{det} H=\left(M_{H} x, x\right)=0$ also follows immediately from Theorem 1 .

To prove Theorem 3 , let $x$ be a unit eigenvector corresponding to the minimum eigenvalue $\lambda$ of $M_{H}$. Thus (4) becomes det $H \leqq \lambda$ and hence $m \operatorname{det} H \leqq \operatorname{tr} M_{H}=d_{\chi}(H)$. If $M_{J} x \neq 0$, then by Theorem 2, $\operatorname{det} H=\lambda \neq 0$ if and only if $H$ is diagonal and nonsingular. If $m \operatorname{det} H$ $=\operatorname{tr} M_{H} \neq 0$, then since $\lambda \geqq \operatorname{det} H$ we must have $\lambda=\operatorname{det} H \neq 0$ and hence $H$ must be diagonal. Conversely, if $H$ is diagonal and nonsingular, then so is $M_{H}$ with diagonal entries $M_{H}(i, i)=\operatorname{det} H, i=1, \cdots, m$. Thus $m \operatorname{det} H=\operatorname{tr} M_{H} \neq 0$ if and only if $H$ is diagonal and nonsingular. If $m \operatorname{det} H=\operatorname{tr} M_{H}=0$, then $\operatorname{det} H=\lambda=0$ and $H$ has a zero row. The converse follows immediately. 


\section{REFERENCES}

1. M. Marcus, On two classical results of I. Schur, Bull. Amer. Math. Soc. 70 (1964), 685-688.

2. M. Marcus and M. Newman, The permanent function as an inner product, Bull. Amer. Math. Soc. 67 (1961), 223-224.

3. I. Schur, Über endliche Gruppen und Hermitesche Formen, Math. Z. 1 (1918), 184-207.

University of California at San Diego, la Jolla 